\title{
Définition de conditions expérimentales pour l'étude des besoins nutritionnels du lapin angora : nature de la litière et présentation du lest alimentaire
}

\author{
J. ROUGEOT, M. COLIN* (1), R. ('. THÉBAULT* \\ avec la collaboration technique de M. BONNET \\ Laboratoire des Pelages, Toisons et Fourrures, \\ Centre national de Recherches Zootechniques, I.N.R.A., \\ 78350 Jouy-en-Josas (France) \\ ** Laboratoire de Recherches sur l'Elevage du Lapin, B.P. I2 \\ Centre de Recherches de Toulouse, I.N.R.A., \\ $3^{1} 3^{20}$ Castanet-Tolosan (France)
}

\begin{abstract}
Résumé
I.e lapin angora ne peut être élevé que sur litière, en raison notamment de la fragilité dé la peau de ses pattes, les sols durs comme le grillage métallique provoquant des escarres qui affectent l'état général et réduisent la production de poil de $25 \mathrm{p}$. I oo. Il en résulte que dans les essais sur l'influence de l'alimentation sur la croissance du poil, qui s'étendent sur au moins un cycle, soit 3 mois, il est nécessaire d'une part de disposer d'une litière non consommable, d'autre part de remplacer le lest fourni par la litière de paille utilisée habituellement.

Trois lots de i I lapines angora adultes, placées sur litière de copeaux de bois, ont reçu pendant deux cycles de croissance du poil, en plus de la ration d'aliment aggloméré commune :

- lot I: $120 \mathrm{~g} / \mathrm{semaine} /$ lapine de paille broyée incorporée dans l'aliment aggloméré;

- lot 2 : la même quantité en fétus dans le ratelier;

- lot 3: paille en fétus ad libilum en ratelier,

les quantités consommées étant contrôlées. Un $4^{\mathrm{e}}$ lot identique, témoin, était élevé sur litière de paille dont la consommation est incontrôlable.

On n'a observé aucun cas pathologique en relation avec la litière ou l'alimentation. Aucune différence significative n'est apparue entre les lots dans la production de poil, qui fut normale (I65 à I $85 \mathrm{~g} /$ animal l'été; 2 ro à $230 \mathrm{~g} /$ animal à 1a récolte d'automne) et qui fut donc indépendante de la forme sous laquelle la paille a été offerte et des quantités ingérées malgré de très grandes variations individuelles $(3,5$ à $43,0 \mathrm{~g} /$ jour pour les cas extrêmes du lot ad libitum).

Comme la consommation de copeaux de bois a été nulle - analyse des fèces après marquage de l'aliment en insoluble chlorhydrique - en raison sans doute de la proportion relativement élevée de lest offerte, de l'ordre de 17 à $18 \mathrm{p}$. I oo, on en conclut qu'il est possible d'entreprendre des expériences de nutrition chez le lapin angora en utilisant une litière faite de ce matériau et avec de l'aliment aggloméré uniquement, pourvu qu'il contienne un lest suffisant.
\end{abstract}

(1) Adresse actuelle : Établissement SANDERS, 17, quai de 1'Industrie, 91200 Athis-Mons. 


\section{Introduction}

Le lapin angora, producteur de poil, est généralement une femelle adulte ayant passé l'âge de 8 mois. La $3^{\mathrm{e}}$ récolte de poil (celle qui commence à compter) se place en effet à cet âge. Le pelage définitif est alors constitué et le poids final de l'animal (4 à $4,5 \mathrm{~kg}$ ) à peu près définitivement établi.

La femelle fournit une toison abondante, plus fine et plus longue que celle du mâle. Chez les sujets sélectionnés, sa production atteint ou dépasse I $\mathrm{kg}$ net de poil par an (les récoltes étant espacées de roo jours environ). On conçoit donc qu'il soit nécessaire de connaître exactement les besoins nutritionnels d'animaux exportant une telle quantité de kératine par rapport à leur poids, d'autant plus que les connaissances sur les besoins nutritionnels spécifiques du lapin angora sont non seulement rares (méthionine, SchloLAUT, I976) mais en outre souvent imprécises. Cela tient à ce que le lapin angora, en production de poil, est un animal adulte et même âgé, et que les besoins nutritionnels ont été surtout déterminés chez le lapin en croissance (CHEEKE, I97I; LEBAS, I975c; CoLIN, I978; CoLIN et AliaAin, i978; Spreadbury et Davidson, 1978) et la lapine en reproduction (LEBAS, 1975b).

Cela est dû surtout à la difficulté, sinon l'impossibilité, de réaliser des essais alimentaires de longue durée sur plancher grillagé avec cette race en raison de la fragilité particulière de la peau des pattes : on observe rapidement, en effet, l'apparition d'escarres sur la face inférieure de celles-ci lorsque le lapin angora est maintenu sur un sol dur. Les troubles pathologiques qui en résultent provoquent, en moyenne, une baisse de $25 \mathrm{p}$. Ioo de la production de poil. Il semble même que les rares individus indemnes d'escarres voient leur production poilière diminuer, vraisemblablement en conséquence d'un confort insuffisant.

Pour réaliser des expériences de nutrition, on est donc obligé de maintenir le lapin angora sur une litière aussi confortable que celle utilisée traditionnellement par les éleveurs.

Or, cette litière est composée de paille que les lapins ingèrent en quantités très variables selon les individus (LEBAS, I 975a) et qu'il est impossible de contrôler.

Nous avons donc envisagé la possibilité de remplacer la paille de la litière par du copeau de bois.

Toutefois, de nombreux travaux réalisés chez le lapin de chair montrent qu'un certain taux de cellulose dans la ration est indispensable à la prévention des troubles digestifs (LEBAS, I975a; Spreadbury et Davidson, I978; Franck et Coulmin, I978).

Dans le cas de lapins élevés sur copeaux de bois, on peut apporter la cellulose par incorporation de paille dans l'aliment granulé, ou éventuellement par distribution de cette matière première sous forme de fétus en plus de la ration de base.

\section{1. - Matériel et méthode}

\section{I.I. - Animaux}

Quarante-quatre lapines angora âgées de 8 ou de II mois (âge auquel on dispose de références sur leur potentiel de production poilière puisqu'elles en 
sont à leur $3^{\mathrm{e}}$ ou $4^{\mathrm{e}}$ récolte) sont réparties en 4 lots en tenant compte des critères suivants présentés par ordre d'importance décroissant : parenté, production poilière, âge, poids vif.

Les lapines sont élevées en clapier individuel traditionnel en ciment; la trémie à granulés et le ratelier de paille sont fixés sur la porte pour faciliter la distribution et le contrôle des aliments.

L'eau de boisson est fournie ad libitum par un abreuvoir à poussoir fixé également sur la porte dans un logement faisant saillie à l'extérieur (de façon à éviter que les fuites d'eau ne mouillent la litière). Les clapiers sont placés dans la lapinerie du laboratoire, consistant en un bâtiment clos où la température ne descend jamais en dessous de Io ${ }^{\circ} \mathrm{C}$. L'éclairage artificiel est programmé pour suivre la variation de la photopériode régnant à la latitude de $45^{\circ}$ Nord.

L'expérience dure pendant 2 périodes successives de croissance du poil, aboutissant aux récoltes d'été et d'automne, soit 2 cycles de 98 jours compris entre le 7 juin et le 2 I décembre. Les lapines sont pesées tous les mois.

\section{r.2. - Traitements expérimentaux (tabl. I)}

\section{I.2I. Principe des essais}

Les lapines mises en expérience sont réparties entre 4 traitements. Les animaux affectés aux traitements $I, 2$ et 3 sont miaintenus sur litière de copeaux.

\section{TABLEAU I}

Traitements expérimentaux sur les 4 lots de II lapins Angora adultes Experimental treatments of the 4 groups of II adult Angora rabbits

\begin{tabular}{|c|c|c|c|c|c|}
\hline & $\begin{array}{l}\text { Numéro } \\
\text { traitement } \\
\text { Treatment } \\
\quad \text { No. }\end{array}$ & & $\begin{array}{c}\text { Type de litière } \\
\text { Litter }\end{array}$ & $\begin{array}{l}\text { Mode de distribution } \\
\text { de la paille } \\
\text { Presentation of the straw }\end{array}$ & $\begin{array}{l}\text { Type et quantité } \\
\text { de granulé distribué } \\
\text { Type and quantity } \\
\text { of pelleted food }\end{array}$ \\
\hline I & . . . . . . &. & $\begin{array}{l}\text { Copeaux de bois } \\
\text { (Wood chips) }\end{array}$ & $\begin{array}{l}\text { Broyée et incorporée dans le gra- } \\
\text { nulé (Io p. Ioo) } \\
\text { (Straw incorporated into the pel- } \\
\text { lets) }\end{array}$ & $\underset{(g / \text { week })}{\mathbf{A}\left({ }^{1}\right)}$ \\
\hline 2 & $. \quad . \quad . \quad$. &. & $\begin{array}{l}\text { Copeaux de bois } \\
\text { (Wood chips) }\end{array}$ & $\begin{array}{l}\text { Distribuée en ratelier en quan- } \\
\text { tité limitée (I } 20 \mathrm{~g} / \text { /semaine) } \\
\text { (Chaffed straw prescnted in racks } \\
\text { in limited amounts) }\end{array}$ & 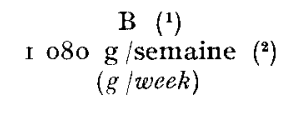 \\
\hline 3 & . . . . . . & $\cdot$. & $\begin{array}{l}\text { Copeaux de bois } \\
\text { (Wood chips) }\end{array}$ & $\begin{array}{l}\text { Distribuée en ratelier ad libitum } \\
\text { (Chaffed straw presented in racks } \\
\text { ad libitum) }\end{array}$ & $\begin{array}{c}\mathrm{B}\left({ }^{1}\right) \\
\mathrm{r} 080 \mathrm{~g} / \text { semaine }\left({ }^{2}\right) \\
(g / w e e k)\end{array}$ \\
\hline &... .5 & $\cdot$. & $\begin{array}{l}\text { Paille } \\
\text { (Straw) }\end{array}$ & $\begin{array}{l}\text { Litière (ad libitum) } \\
\text { (Litter (ad libitum) }\end{array}$ & $\begin{array}{c}\mathrm{B}\left({ }^{1}\right) \\
\text { I } 080 \mathrm{~g} / \text { semaine }\left({ }^{2}\right) \\
(g / \text { week })\end{array}$ \\
\hline
\end{tabular}

(1) Aliment $\mathrm{A}=$ ro p. 100 de paille (Pellets $A=I 0$ p. roo straw). Aliment $\mathrm{B}=0$ p. roo de paille (Pellets $B=o$ p. Ioo strai ).

(2) Distribués pendant les 6 premiers jours de la semaine (pas de distribution d'aliment le dimanche) (Offered during the first 6 days of the week (no feeding on Sundays)). 
On leur apporte alors de la paille de blé alimentaire, soit en l'incorporant dans la ration (traitement $I$ ), soit en la distribuant dans un ratelier en quantités rationnées (traitement 2) ou à volonté (traitement 3).

Enfin, les lapines correspondant aı traitement 4 sont élevées dans des conditions traditionnelles sur litière de paille de blé.

\section{I.22. Litière}

Les lapines des lots expérimentaux I, 2 et 3 (différant uniquement par la modalité d'apport de paille) sont élevées sur litière de copeaux de bois, dépoussiérés au tarrare. Par contre, celles du lot 4 sont maintenues sur litière traditionnelle de paille de blé. Les copeaux de bois dont des fragments de $1 / 2$ à $2 \mathrm{~cm}^{2}$ constituant un mélange de bois blanc, de résineux et de chêne. La paille de blé est prélevée dans un stock réservé de balles compressées et se présente sous forme de fétus de Io à $30 \mathrm{~cm}$ de long. Le stock fournit également la paille alimentaire des autres lots.

Les litières de paille ou de copeaux de bois sont évacuées entièrement tous les mois; un apport de paille ou de copeaux de bois (toujours plus de $200 \mathrm{~g}$ ) est effectué toutes les semaines.

Afin de vérifier la non ingestion des copeaux de bois par les lapins, nous comparons la teneur de l'aliment et des fèces en lignine et en insoluble chlorhydrique.

Pour réaliser cette étude, l'aliment est enrichi en insoluble chlorhydrique par introduction de $0,5 \mathrm{p}$. Ioo de célite 425 . Les prélèvements de fèces destinés à ce travail commencent 13 jours après distribution de la ration contenant la célite 425 ; ils sont renouvelés chaque matin pendant I I jours consécutifs chez les lapins des lots $n^{\text {os }} \mathrm{r}, 2$ et 3 .

\section{I.23. Régimes alimentaires}

On utilise 2 régimes expérimentaux se différenciant par leur taux de paille (Io p. Ioo pour l'aliment A, o p. Ioo pour l'aliment B) (tab1. 2). La ration B (dont la formule correspond à celle utilisée avec succès depuis plusieurs années dans l'élevage) est distribuée à raison de $\mathrm{I} 80 \mathrm{~g} / \mathrm{j}$ pendant les 6 jours de la semaine (les animaux ne recevant rien le dimanche). La ration $A$ (contenant ro p. Ioo de paille) est apportée à raison de $200 \mathrm{~g} / \mathrm{j}$ également pendant les 6 jours de la semaine. De la sorte, les animaux ingèrent la même quantité d'aliment autre que la paille dans les 4 traitements.

Dans les lots 2 et 3 , les animaux reçoivent, en plus du régime granulé, de la paille en fétus, de $\mathrm{r} 0$ à $30 \mathrm{~cm}$ de longueur, soit à raison de $20 \mathrm{~g} / \mathrm{j}$ (quantité analogue à celle consommée par les animaux recevant la ration avec ro p. Ioo de paille) (lot 2), soit ad libitum (lot 3).

\section{I.24. Mesure de la production de poil}

Les récoltes de poil sont effectuées par épilation après traitement au cyclophosphamide pour faciliter l'opération et éviter le stress (Rougeor et Thíbaur,T, 1976). Ainsi, les lapines sont toutes épilées le 7 juin, jour de la mise en place de l'expérience. La $\mathrm{I}^{\mathrm{re}}$ récolte de la période expérimentale a lieu 98 jours (I 4 semaines) après le début de l'expérience (le $\mathrm{I} 4$ septembre) et la $2^{\mathrm{e}}, 9^{8}$ jours plus tard (le 2 I décembre). Les pesées des toisons sont effectuées au gramme près. 


\section{TABLEAU 2}

Composition des régimes alimentaires (en p. Ioo de la matière sèche)

Composition of diets (in p. Ioo of dry matter)

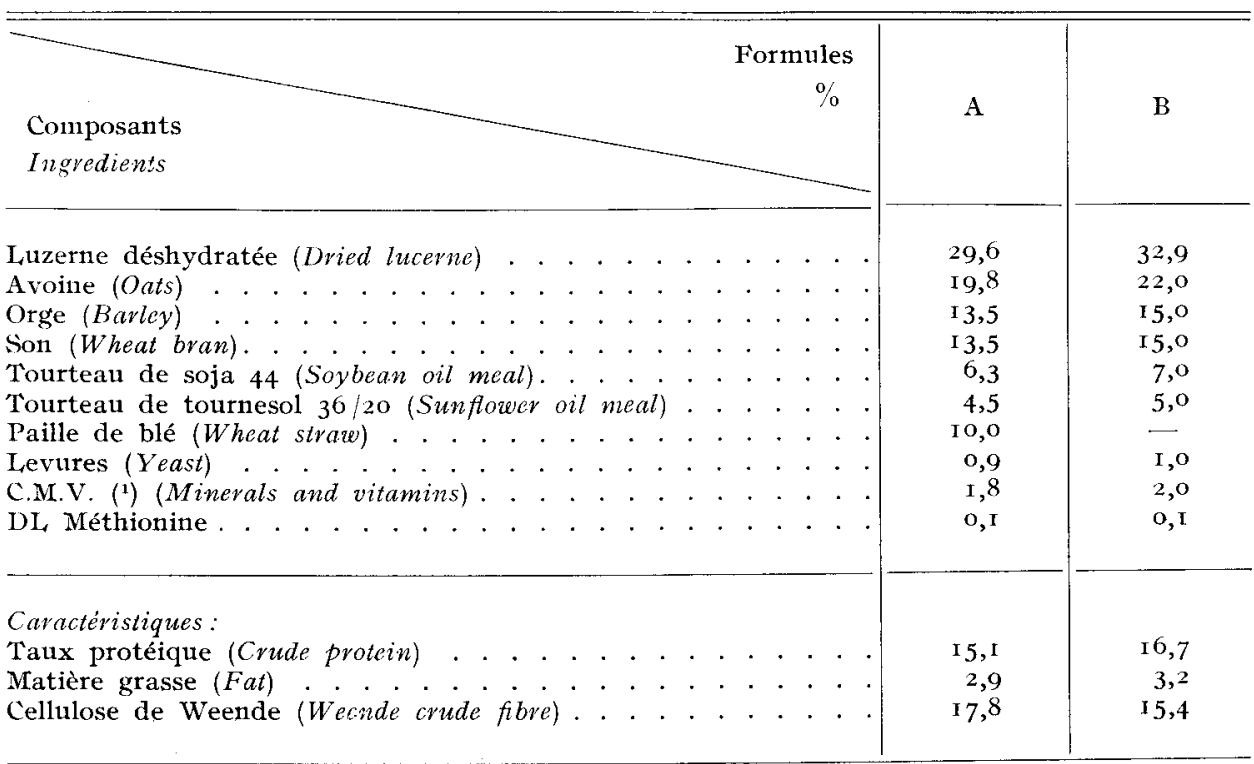

(1) Composition :

Minéraux (en p. 100 du C.M.V.) :

- Phosphate monocalcique. . . . . . . . . . . 59,4I

- Chlorure de sodium . . . . . . . . . . . . 24,74

- Carbonate de magnésium . . . . . . . . . . . 13,65 $^{3}$

- Sulfate de fer . . . . . . . . . . . . . 0,531

- Sulfate de manganèse . . . . . . . . . . 0,976

- Sulfate de cobalt. . . . . . . . . . . . . 0,00r

- Sulfate de cuivre. . . . . . . . . . . . . . . 0,099

- Sulfate de zinc . . . . . . . . . . . . . . 0,593

Vitamines (pour $100 \mathrm{~kg}$ d'aliment) :

- Vitamine A. . . . . . . . . . . . I 000 o0o U.I.

- E.................... $5000 \mathrm{mg}$

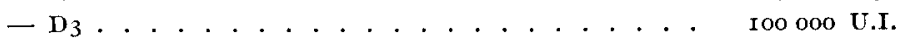

\section{I.3. - Méthodologie statistique}

L'exploitation statistique des résultats est assurée par 1'analyse de variance pour les différents traitements, sauf pour les taux de paille ingérée qui sont comparés à l'aide du test de KRUSKALL et WALLIS (DAGNELIE, I975). Les différences d'une période à l'autre sont étudiées par la méthode des couples. 


\section{2. - Résultats}

\section{I. - État général des lapins}

La mortalité (I mort dans le lot I et 2 dans chacun des 3 autres lots) apparaît normale compte tenu de l'effectif (44) et de la durée de l'expérience (6 mois $1 / 2$ ).

En outre, cette mortalité est à mettre en rapport avec des entités pathologiques considérées comme classiques chez le lapin angora (RENAULT, I975; BRU, I975; RIVE, I975).

\section{2. - Consommation de copeaux de bois (tabl. 3)}

Les rapports entre la teneur en lignine et la teneur en insoluble chlorhydrique sont pratiquement identiques pour l'aliment et les fèces (tab1. 3). Par contre, les copeaux de bois utilisés pour la litière sont très riches en lignine et pauvres en insoluble chlorhydrique.

Dans ces conditions, l'ingestion éventuelle d'une quantité, même faible, de copeaux augmenterait l'excrétion fécale de lignine et conduirait donc à un rapport

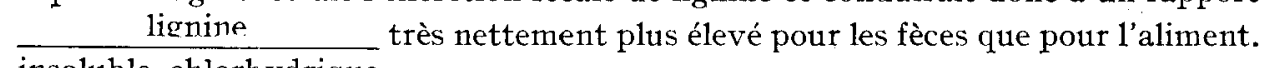
insoluble chlorhydrique

Un tel phénomène n'étant pas observé, on peut en déduire que l'ingestion de copeaux de bois pour les animaux est restée à un niveau nul ou négligeable.

\section{TABI,EAU 3}

Teneur en lignine et insoluble chlorhydrique de l'aliment, des fèces et des copeaux de bois

Lignin content and $4 \mathrm{~N} \mathrm{HCl}$ insoluble ashes in feed, faeces and wood chips

\begin{tabular}{|c|c|c|c|}
\hline & $\begin{array}{c}\text { Lignine } \\
\text { (p. too produit frais) } \\
\text { Lignin } \\
\text { (p. Ioo frosh focd) }\end{array}$ & $\begin{array}{c}\text { Insoluble } \\
\text { chlorhydrique (I.C.) } \\
\text { (p. Ioo produit frais) } \\
\mathrm{HCl} \text { insoluble ashes } \\
(p . \text { Ioo fresh feed) }\end{array}$ & $\begin{array}{c}\text { Rapport lignine I.C. } \\
\text { Lignin } / F C \text { ratio }\end{array}$ \\
\hline Aliment $(F e c d)$. & 4,29 & $I, 56$ & 2,75 \\
\hline Fèces (Faeces) & $6,67 \pm 0,12\left(^{1}\right)$ & $2,45 \pm 0,02$ & $2,71 \pm 0,05$ \\
\hline Copeaux (Wocd chips). & 25,9 & 0,1 & 259,0 \\
\hline
\end{tabular}

(1) Écart-type de la moyenne des ir jours de prélèvement (S.E.M., II days of sampling). 


\section{3. -- Consommation d'aliment}

\subsection{Aliment granulé (tabl. 4)}

Si l'on considère la fraction de la ration ne comportant pas de paille (granulé $B$ et $90 \mathrm{p}$. Ioo du granulé A), on constate que les consommations sont identiques pendant les 2 périodes et pour les 4 lots (phénomène paraissant normal compte tenu du rationnement imposé aux animaux). Pour l'ensemble de la $x^{\text {re }}$ période, la consommation reste comprise entre $\mathrm{I} 45$ et $\mathrm{I} 49( \pm 2)$ et pour la $2^{\mathrm{e}}$ période entre $\mathrm{I}_{50}$ et $\mathrm{I}_{52}( \pm 2) \mathrm{g} / \mathrm{j}$ (différences non significatives).

Il faut cependant noter que si le granulé distribué est (à de très rares exceptions près) intégralement consommé pendant les 2 mois suivant chacune des récoltes de poil, on enregistre de nombreux refus au cours du $3^{\mathrm{e}}$ mois.

Ce phénomène est particulièrement marqué pour la I $^{\mathrm{r}}$ période expérimentale de récolte (- I3 p. roo par rapport aux 2 premiers mois) mais se retrouve également pendant la $2^{\mathrm{e}}(-4,5$ p. Ioo $)$.

\subsection{Paille}

Les lapines disposant de fétus de paille ad libitum dans le ratelier (lot 3) ingèrent en moyenne autant de paille que celles du lot I où la paille est incluse dans le granulé, avec toutefois des variations individuelles considérables.

\section{TABLEAU 4}

Consommation moyenne quotidienne en aliments agglomérés ( $g / j /$ lapin)

Daily mean intake of pellets ( $g /$ day/rabbit)

\begin{tabular}{|c|c|c|c|c|c|c|}
\hline & & Lot I (') & Lot 2 & Lot 3 & Lot 4 & $\begin{array}{l}\text { Signification } \\
\text { statistique } \\
\text { Level } \\
\text { of significance }\end{array}$ \\
\hline \multirow[t]{2}{*}{$\begin{array}{c}\text { Été } \\
\text { Summer }\end{array}$} & $\begin{array}{l}\mathrm{I}^{\mathrm{er}} \text { mois }(\text { month) } \\
2^{\mathrm{e}} \text { mois } . . \\
3^{\mathrm{e}} \text { mois } \cdot . \cdot .\end{array}$ & $\begin{array}{l}\text { I } 54 \\
\text { I } 54 \\
\text { I } 34\end{array}$ & $\begin{array}{l}\text { I } 54 \\
\text { I } 54 \\
\text { I } 39\end{array}$ & $\begin{array}{l}\text { I } 54 \\
\text { I } 54 \\
\text { I } 27\end{array}$ & $\begin{array}{l}\text { I } 54 \\
\text { I } 54 \\
\text { I } 36\end{array}$ & \\
\hline & $\bar{x} . . . . . . .$. & $147 \pm 2(2)$ & $149 \pm 2$ & I $45 \pm 2$ & I $48 \pm 2$ & NS \\
\hline $\begin{array}{l}\text { Automne } \\
\text { Autumn }\end{array}$ & 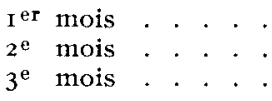 & $\begin{array}{l}154 \\
152 \\
146\end{array}$ & $\begin{array}{l}\text { I } 53 \\
\text { I } 54 \\
\text { I } 48\end{array}$ & $\begin{array}{l}\text { I } 54 \\
\text { I } 54 \\
\text { I } 46\end{array}$ & $\begin{array}{l}148 \\
\text { I } 54 \\
\text { I } 50\end{array}$ & \\
\hline & 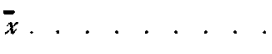 & I $50 \pm 2$ & $152 \pm 2$ & I5I \pm 2 & ${ }^{15} \mathrm{I} \pm 2$ & NS \\
\hline
\end{tabular}

(1) La quantité de paille incluse dans le granulé a été déduite pour la comparaison (The amount of straw incorporated into pellets $A$ is deduced for comparison between groups).

( ${ }^{2}$ ) Écart-type de la moyenne (S.E.M.). 
Ainsi, on enregistre les valeurs extrêmes suivantes : pour une lapine, 38 , r et $43,0 \mathrm{~g} / \mathrm{j}$ respectivement pendant la $\mathrm{I}^{\mathrm{re}}$ et la $2^{\mathrm{e}}$ période; pour une autre lapine, respectivement 5,5 et $3,5 \mathrm{~g} / \mathrm{j}$.

Comme pour l'aliment granulé, on assiste à un abaissement de la consommation de paille pendant le $3^{e}$ mois suivant une épilation. Par rapport aux résultats des 2 premiers mois, cette diminution est de 28 p. Ioo pendant la ${ }^{r e}$ période et $14,5 \mathrm{p}$. Ioo pendant la $2^{\mathrm{e}}$.

E,n outre, dans le lot 2 , la paille distribuée à raison de $20 \mathrm{~g} / \mathrm{j}$ n'est qu'exceptionnellement consommée en totalité (tabl. 5).

\section{TABLEAU 5}

Consommation moyenne quotidienne de paille en valeur absolue ( $\mathrm{g} / \mathrm{j} / \mathrm{lapin}$ ) et en pourcentage par rapport aux quantités totales d'aliments ingérés

Daily mean intake of straw in absolute value (g/day/rabbit) and in per cent of the total level of feed intake

\begin{tabular}{|c|c|c|c|c|c|c|}
\hline & & Lot I & Lot 2 & Lot 3 & Lot 4 & $\begin{array}{l}\text { Signification } \\
\text { statistique } \\
\text { Level } \\
\text { of significance }\end{array}$ \\
\hline $\begin{array}{c}\text { Eité } \\
\text { Summer }\end{array}$ & $\mid \begin{array}{lll}\mathrm{I}^{\mathrm{er}} & \text { mois }(\text { month }) \\
2^{\mathrm{e}} & \text { mois } . & . \\
3^{\mathrm{e}} & \text { mois } . & .\end{array}$ & $\begin{array}{l}\text { I } 7, \text { I } \\
\text { I } 7, \mathrm{I} \\
\text { I } 4,3\end{array}$ & $\begin{array}{l}\text { I } 5,2 \\
13,3 \\
\text { I } 0,4\end{array}$ & $\begin{array}{r}19,0 \\
14,9 \\
9,8\end{array}$ & $\begin{array}{l}(1) \\
(1) \\
(1)\end{array}$ & \\
\hline & $\bar{x} . . . . . .$. & $16,3 \pm 0,2\left({ }^{3}\right)$ & $13,2 \pm 0,6$ & $\mathrm{I} 4,9 \pm 3, \mathrm{I}$ & (1) & NS \\
\hline $\begin{array}{l}\text { Automne } \\
\text { Autumn }\end{array}$ & $\mid \begin{array}{lllll}\text { I }^{\mathbf{e r}} & \text { mois } & . & . & . \\
2^{\mathbf{e}} & \text { mois } & . & . & . \\
3^{\mathrm{e}} & \text { mois } & . & . & .\end{array}$ & $\begin{array}{l}\text { I } 7, \text { I } \\
\text { I } 6,8 \\
\text { I } 6,2\end{array}$ & $\begin{array}{l}\text { I } 2,6 \\
\text { I } 1,6 \\
\text { I } 0,9\end{array}$ & $\begin{array}{l}\text { I } 9,2 \\
\text { I } 7,7 \\
\text { I } 3,6\end{array}$ & $\begin{array}{l}(1) \\
\left({ }^{1}\right) \\
\left({ }^{1}\right)\end{array}$ & \\
\hline & $\bar{x} . . . . .$. & I $6,7 \pm 0,2$ & $\mathrm{I} \mathrm{I}, \mathrm{B} \pm \mathrm{I}, \mathrm{O}$ & I $6,7 \pm 3,8$ & (1) & NS \\
\hline $\begin{array}{c}\text { Taux } \\
\text { de paille }\end{array}$ & Été. . . . & Io & $8,0 \pm 0,3$ & $8,6 \pm \mathrm{I}, 5$ & (1) & $*(2)$ \\
\hline of slvaw) & Automne . . & IO & $7,2 \pm 0,5$ & $9,5 \pm 1,8$ & (1) & $*(2)$ \\
\hline
\end{tabular}

(1) Donnée non mesurable (Non measurable data).

(2) Différence statistique au seuil $\mathrm{P}<0,05\left(P<.0_{5}\right)$.

( $\left.{ }^{3}\right)$ Écart-type de la moyenne (S.E.M.).

\section{4. - Production de poil (tabl. 4)}

Il n'existe aucune différence significative entre la production de poils correspondant aux 4 lots (et ceci quelle que soit la saison considérée). Par contre, la production poilière est significativement plus élevée en automne dans chacun des 4 lots. 


\section{TABLEAU 6}

Moyenne de la production de poils des récoltes d'été et d'automne. portant chacune sur 98 jours de croissance de la toison

Mean of Angora rabbit wool production in Summer and Autumn harvestings after 98 days fibre growth

\begin{tabular}{|c|c|c|c|c|c|c|c|}
\hline & $\begin{array}{c}\text { Traitements }\left({ }^{1}\right) \\
\text { Treatments } \\
\text { Période } \\
\text { Period }\end{array}$ & \begin{tabular}{|c|}
$\mathbf{I}$ \\
Paille \\
dans \\
granulé \\
Straw \\
in $\begin{array}{l}\text { pelleted } \\
\text { feed }\end{array}$
\end{tabular} & $\begin{array}{c}2 \\
\text { Paille } \\
\text { dans } \\
\text { ratelier } \\
\text { rationnée } \\
\text { Straw } \\
\text { in racks } \\
\text { restricted } \\
\text { amount }\end{array}$ & $\begin{array}{c}3 \\
\text { Paille } \\
\text { dans } \\
\text { ratelier } \\
\text { ad libitum } \\
\text { Straw } \\
\text { in racks } \\
\text { ad libitum }\end{array}$ & $\begin{array}{c}4 \\
\text { Paille } \\
\text { en litière } \\
\text { Litter } \\
\text { straw } \\
\text { only }\end{array}$ & $\begin{array}{r}\text { Significa } \\
\text { statistiqu } \\
\text { Leve } \\
\text { of signifi }\end{array}$ & $\begin{array}{l}\text { tion } \\
\text { le }\left({ }^{2}\right) \\
l \\
\text { cance }\end{array}$ \\
\hline \multirow{2}{*}{$\begin{array}{l}\text { Production } \\
\text { de poil } \\
\text { (g/lapin) } \\
\text { Wool } \\
\text { production } \\
\text { (g/rabbit) }\end{array}$} & Été (Summev). . . & $172 \pm 7$ & I $64 \pm$ Io & $180 \pm 13$ & $185 \pm 7$ & $\mathrm{~T}: \mathrm{NS}$ & \multirow[b]{2}{*}{$\mathrm{P}^{* *}$} \\
\hline & Automne (Autumn). & $227 \pm 4$ & $23^{\circ} \pm 9$ & $210 \pm 14$ & $227 \pm 8$ & $\mathrm{~T}: \mathrm{NS}$ & \\
\hline
\end{tabular}

(1) II lapins angora par traitement ( $I I$ rabbits in each treatment).

(2) $\mathrm{T}$ : Effet traitement (Treatment efect).

$P$ : Effet période (Period effect)

NS : Non significatif.

* Significatif au seuil $\mathbf{P}=0,05(P<.05)$.

** Significatif au seuil $\mathrm{P}=0,0$ I $(P<. O I)$.

\section{Discussion}

Il ressort des résultats présentés ici que l'utilisation de litières à base de copeaux de bois convient parfaitement pour réaliser des expériences nutritionnelles sur lapins angora. En effet, l'état général des animaux s'avère satisfaisant pour les 4 traitements (en particulier, ancune des 33 lapines maintenues sur litière de copeaux ne présente d'escarres) et la production de poils est normale et identique pour les 4 lots. La nature de la litière n'affecte pas l'aspect général et la propreté de la toison si l'on excepte le fait que les copeaux s'accrochent au poil, nécessitant un brossage avant épilation (opération laborieuse pour des éleveurs, mais peu gênante au cours d'une expérimentation).

En outre, on peut affirmer que les animaux n'ont pas consommé les copeaux de la litière puisque le rapport insoluble chlorhydrique est identique dans l'aliment et les fèces.

I1 faut toutefois préciser que ces observations sont réalisées pour des lapins dont le régime contient des quantités très importantes de cellulose : en effer, le taux de cellulose de l'aliment granulé de base est de $15,4 \mathrm{p}$. Ioo; si l'on ajoute à ce taux la paille incorporée dans l'aliment ou distribuée en fétus, on en déduit que le niveau de cellulose du mélange alimentaire consommé par les animaux 
est compris entre I7,o et I 8,0 p. Ioo, taux nettement supérieur au niveau d'apport minimal de cellulose dans les aliments pour lapins de chair : I4,0 p. roo d'après Lebas (I975), Spreadbury et Davidson (I978), Franck et Coulmin (I978).

Dans ces conditions, il paraît normal que les animaux ne recherchent aucune source de lest autre que la ration de base ou la paille et qu'ils ne consomment pas de copeaux.

Par ailleurs, au cours de cet essai, on observe que les quantités de poils récoltées sont plus fortes pendant la période d'automne que pendant la période d'été. Cette variation saisonnière chez le lapin angora adulte a déjà été constatée par ThúBAuLT (1977); elle est très vraisemblablement liée au photopériodisme saisonnier.

De même, on enregistre des consommations de granulés un peu plus élevées en automne qu'en été. Il serait nécessaire, pour interpréter cette évolution, de disposer de références concernant les autres saisons.

Enfin, on remarque que, pendant le $3^{\mathrm{e}}$ mois suivant une épilation, l'ingestion de paille et d'aliment diminue très sensiblement: I3 et 4,5 p. Ioo respectivement pour l'alinient au cours de la ${ }^{\mathrm{re}}$ et $1 \mathrm{a} 2^{\mathrm{e}}$ période; 28 et $14,5 \mathrm{p}$. Ioo pour la paille.

Il faut mettre cette diminution en relation avec l'ingestion d'une quantité appréciable de poils pendant ce $3^{\mathbf{e}}$ mois, phénomène pouvant même aboutir à la formation de trichobezoards (Rovgeot et ThÉBAuL'T, r977).

\section{Conclusion}

En conclusion, il est possible, pour réaliser des essais nutritionnels, de maintenir des lapins angora sur litières de copeaux de bois. Dans les conditions de l'essai, ce matériau n'est pas ingéré par les lapins et il procure aux animaux le confort exigé. Pour éliminer les risques de consommiation de copeaux de bois et éviter toute variation incontrôlable de l'ingestion de paille, la solution la plus simple pour le moment consiste à distribuer un aliment très riche en cellulose (17 à I 8 p. roo); l'obtention de taux de cellulose aussi élevés est réalisée par incorporation de ro p. Ioo de paille dans le granulé.

Grâce à la méthodologie ainsi mise au point, il apparaît possible de procéder à l'étude des besoins alimentaires du lapin angora.

Accepté pour publication en décembre r979.

\section{Summary}

Experimental conditions for studying the nutritional requirements of angora rabbits: nature of the litter and physical form of the bulk

Angora rabbits have to be raised on a litter, because hard and rigid floors are uncomfortable and quickly produce eschars on the paws which may affect the general condition of the animals and cause a 25 p. Ioo decrease in the amount of wool harvested. As it is necessary to use nonedible litters when studying the influence of nutritional factors on the Angora wool growth which last 3 months, we tested litters made of wood chips ( $4 \mathrm{~N} \mathrm{HCl}$ insoluble ashes test, table 3 ) 
and an adequate supply of bulk (I 7-I $8 \mathrm{p}$. roo) given with the food (pellets). Observations were made on 4 groups of I adult females (table I) fed on a common basal diet (table 2) supplemented with different forms and amounts of wheat straw:

1) I $20 \mathrm{~g} / \mathrm{week} / \mathrm{rabbit}$ of ground straw incorporated into the pellets;

2) the same quantity of chaffed straw presented in racks;

3) chaffed straw presented ad libitum in racks and

4) litter straw only.

The first 3 groups were kept on litters made of wood chips. No pathological event could be attributed to the litter or diet. No significant differences were observed between groups in the wool production which was normal ( $165^{-1} 85 \mathrm{~g} / \mathrm{rabbit}$ in Summer; $210-230 \mathrm{~g} / \mathrm{rabbit}$ in Autumn; table 6) and independent of the form and the level of intake of wheat straw (table 5), in spite of large individual differences between animals $(3.5$ to $43.0 \mathrm{~g} / \mathrm{day}$ for the extreme cases in the ad libitum group).

\section{Références bibliographiques}

BRU C., I975. Pathologie respiratoire du lapin. Inf. tech. Dir. Serv. Vét., no 5 I à 54, spécial "Le lapin ", I 24-128.

CHEFkE P. R., 197I. Arginine, lysine and methionine needs of the growing rabbit. Nutr. Rept. Int., 3, $123-128$.

CoLIN M., I978. Effet d'une supplémentation en méthionine ou en cystine de régimes carencés en acides aminés soufrés sur les performances de croissance du lapin. Ann. Zootech., $27,9^{-16}$.

Colin M., Allain D., I978. Étude du besoin en lysine du lapin en croissance en relation avec la concentration énergétique de l'aliment. Ann. Zootech., 27, I7-3 I.

DAGNEIIE P., I975. Théorie et méthodes statistiques. Applications agronomiques. II. - Ies méthodes de l'interférence statistique. 390-394, $2^{\mathrm{e}}$ édition, Les Presses Agronomiques de Gembloux, Gembloux.

FISHER H., I976. Protein and amino acid requirement of the laboratory rabbit. Lab. anim. Sci., 26, 659-663.

Franck Y., Coutmin J. P., 1978. Utilisation de la paille broyée comme source de cellulose dans les aliments lapins à l'engraissement, comparaison avec deux taux de cellulose. $2^{\mathrm{e}} \mathrm{Jou}$ nées Rech. Cunicoles, Toulouse, 4-5 avril I978, Comm. no ro, 6 pp.

LEBAS F., I975a. Le lapin de chair, ses besoins nutritionnels et son alimcntation, 50 pp., 2e éd., Institut Technique de l'Aviculture, Paris.

LFBAS F., I975 b. Étude chez la lapine de l'in fluence du niveau d'alimentation durant la gestation. I. - Sur les performances de reproduction. Ann. Zootech., 24, 267-279.

LFBAS F., I975c. Influence de la teneur en énergie de l'aliment sur les performances de croissance chez le lapin. Ann. Zootech., 24, 28I-288.

Renauit L., 1975. Pathologie digestive du lapin. Dans "Le lapin : règles d'élevage ct d'hygiène ". Inf. tech. Dir. Serv. Vét., no 5 I à 54 , spécial "Le lapin ", I 29-I 4 I

RIve M., 1975. Le lapin angora. Inf. tech. Dir. Sevv. Vét., $\mathrm{n}^{\circ} \mathbf{5}^{\mathrm{I}}$ à 54 , spécial “ Le lapin ", $163-\mathrm{I} 66$.

Rovgrót J., Thébault R. G., I976. Pratique de la récolte du poil du lapin angora par épilation après traitement au cyclophosphamide. Ier Congrès Int. Cunicole, Comm. $\mathrm{n}^{\circ} 73,4 \mathrm{pp}$.

Rougeot J., Thébault R. G., I977. Formation de trichobézoards chez le lapin angora nourri ad libitum avec un aliment aggloméré. Rec. Méd. Vét., 153, 655-659.

Schlolau' W., 1976. Der Einfluss von Alter, Geschlecht und Fütterung auf die Wolleistung bein Angorakaninchen. I er Congrès Int. Cunicole, Comm. no $19,5 \mathrm{pp}$.

SPREAdBURy D., DAvidson J., I978. Some observations on the arginine requirements of the growing New-Zealand White rabbit. $J$. Sci. Fd. Agric., 29, Ior7-1022.

Sprifadbury D., Davidson J., I978. A study of the need for fibre by the growing New Zealand rabbit. J. Sci. Fd. Agric., 29, 640-648.

ThúbAuLt R. G., 1977. Le lapin angora. Développement post-natal de sa toison; variation saisonniève de sa production de poil. Mém. Ing. D.P.E., Spécialité Agriculture, Conservatoire Nat. Arts et Métiers, Paris, I05 pp. 